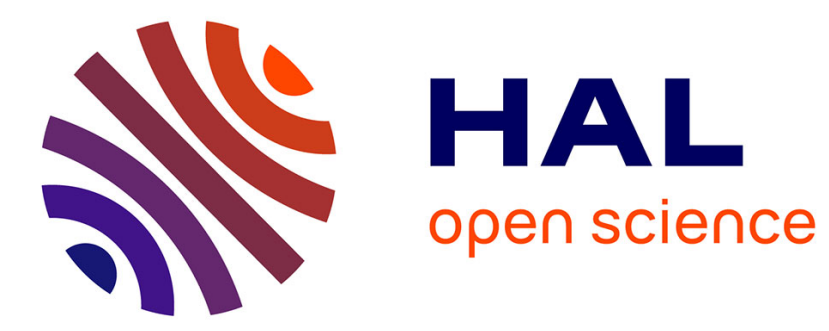

\title{
Logic Fragments: A Coordination Model Based on Logic Inference
}

\author{
Francesco Angelis, Giovanna Di Marzo Serugendo
}

\section{To cite this version:}

Francesco Angelis, Giovanna Di Marzo Serugendo. Logic Fragments: A Coordination Model Based on Logic Inference. 17th International Conference on Coordination Languages and Models (COORDINATION), Jun 2015, Grenoble, France. pp.35-48, 10.1007/978-3-319-19282-6_3 . hal-01774943

\section{HAL Id: hal-01774943 \\ https://hal.inria.fr/hal-01774943}

Submitted on 24 Apr 2018

HAL is a multi-disciplinary open access archive for the deposit and dissemination of scientific research documents, whether they are published or not. The documents may come from teaching and research institutions in France or abroad, or from public or private research centers.
L'archive ouverte pluridisciplinaire $\mathbf{H A L}$, est destinée au dépôt et à la diffusion de documents scientifiques de niveau recherche, publiés ou non, émanant des établissements d'enseignement et de recherche français ou étrangers, des laboratoires publics ou privés. 


\title{
Logic Fragments: a coordination model based on logic inference
}

\author{
Francesco L. De Angelis \\ francesco.deangelis@unige.ch and \\ Giovanna Di Marzo Serugendo \\ giovanna.dimarzo@unige.ch \\ Institute of Information Services Science, University of Geneva, SWITZERLAND
}

\begin{abstract}
Chemical-based coordination models have proven useful to engineer self-organising and self-adaptive systems. Formal assessment of emergent global behaviours in self-organising systems is still an issue, most of the time emergent properties are being analysed through extensive simulations. This paper aims at integrating logic programs into a chemical-based coordination model in order to engineer self-organising systems as well as assess their emergent properties. Our model is generic and accommodates various logics. By tuning the internal logic language we can tackle and solve coordination problems in a rigorous way, without renouncing to important engineering properties such as compactness, modularity and reusability of code. This paper discusses our logic-based coordination model and shows how to engineer and verify a simple pattern detection example and a gradient-chemotaxis example.
\end{abstract}

\section{Introduction}

Coordination models have been proven useful for designing and implementing distributed systems. They are particularly appealing for developing self-organising systems, since the shared tuple space on which they are based is a powerful paradigm to implement self-organising mechanisms, particularly those requiring indirect communication (e.g. stigmergy) [16] Chemical-based coordination models are a category of coordination models that use the chemical reaction metaphor and have proven useful to implement several types of self-organising mechanisms [18]. A well-known difficulty in the design of self-organising systems stems from the analysis, validation and verification (at design-time or run-time) of so-called emergent properties - i.e. properties that can be observed at a global level but that none of the interacting entities exhibit on its own. Few coordination models integrate features supporting the validation of emergent properties, none of them relying on the chemical metaphor. 
In this paper, we propose to enrich a chemical-based coordination model with the notion of Logic Fragments (i.e. a combination of logic programs). Our logic-based coordination model allows agents to inject Logic Fragments into the shared space. Those fragments actually define on-the-fly ad hoc chemical reactions that apply on matching data tuples present in the system, removing tuples and producing new tuples, possibly producing also new Logic Fragments. Our model is defined independently of the logic language used to define the syntax of the Logic Fragment, an actual instantiation and implementation of the model can use its own logic(s). The advent of new families of logic languages (e.g. [17]) has enriched the paradigm of logic programming, allowing, among other things, practical formalisation and manipulation of data inconsistency, knowledge representation of partial information and constraints satisfaction. By combining those logics with a chemical-based coordination model, we argue that global properties can be verified at design time.

Section 2 discusses related works, section 3 presents our logic-based coordination model. Section 4 shows two case studies: a simple pattern recognition example and another one with the gradient and chemotaxis patterns. Finally, section 5 concludes the paper.

\section{Related works}

\subsection{Chemical-based coordination models}

An important class of coordination models is represented by so-called chemical-based coordination models, where "chemical" stands for the process of imitating the behaviours of chemical compounds in chemical systems.

Gamma (General Abstract Model for Multiset mAnipulation) [2] and its evolutions historically represents an important chemical-inspired coordination model. The core of the model is based on the concept of virtual chemical reactions expressed through condition-action rewriting pairs. Virtual chemical reactions are applied on input multisets which satisfy a condition statement and they produce as output multisets where elements are modified according to the corresponding action (like for chemical compounds); the execution of virtual chemical reactions satisfying a condition pair is nondeterministic. Gamma presents two remarkable properties: (i) the constructs of the model implicitly support the definition of parallel programs; (ii) the language was proposed in the context of systematic program derivation and correctness as well as termination of programs is 
easy to prove ([8]). Its major drawback is represented by the complexity of modeling real large applications.

The SAPERE model [4] (Figure 1a) is a coordination model for multiagent pervasive systems inspired by chemical reactions. It is based on four main concepts: Live Semantic Annotations (LSAs), LSA Tuple Space, agents and eco-laws. LSAs are tuples of types (name, value) used to store applications data. For example, a tuple of type (date, 04/04/1988) can be used to define a hypothetical date. LSAs belonging to a computing node are stored in a shared container named LSA Tuple Space. Each LSA is associated with an agent, an external entity that implements some domainspecific logic program. For example, agents can represent sensors, services or general applications that want to interact with the LSA space - injecting or retrieving LSAs from the LSA space. Inside the shared container, tuples react in a virtual chemical way by using a predefined set of coordination rules named eco-laws, which can: (i) instantiate relationships among LSAs (Bonding eco-law); (ii) aggregate them (Aggregate eco-law); (iii) delete them (Decay eco-law) and (iv) spread them across remote LSA Tuples Spaces (Spreading eco-law). Spontaneous executions of ecolaws can be fired when specific commands (named operators) are present in tuple values. When a tuple is modified by an eco-law, its corresponding agent is notified: in this way, agents react to virtual chemical reactions according to the program they implement. The implementation of the SAPERE model, named SAPERE middleware, has been proven to be powerful enough and robust to permit the development of several kinds of real distributed self-adaptive and self-organising applications, as reported in [18]. Nevertheless, the model does not aim at proving correctness or emergence of global properties programs built on it: this means that proving correctness of applications may turn to be a complex task.

\subsection{Formal approaches for tuple based coordination models}

Coordination models based on tuple spaces are amenable to several kinds of analytical formalisation.

PoliS [5] is a coordination model based on multiset rewriting in which coordination rules consume and produce multisets of tuples; rules are expressed in a Chemical Abstract Machine style [3]. In PoliS, properties can be proved by using the PoliS Temporal Logic and the PoliMC model checker.

Tuples centres [15] allow the use of a specification language (named RespecT) to define computations performed in the tuple space. Computations are associated with events triggered internally because of reactions 
previously fired or during the execution of traditional input/output operations by agents. RespecT is based on first-order logic and unification of unitary clauses (tuple templates) and ground atoms (tuples) represent the basic tuple matching mechanism.

In the ACLT model [7], the tuple space is treated as a container of logic theories, which can be accessed by logic agents to perform deduction processes. Again, the first-order logic and unification of unitary clauses and ground atoms is used as matching mechanism; the model offers specific input-output primitives tailored to provide different meaning for unification by allowing a certain control in selecting the set of unitary clauses to be treated as facts in case of backtracks or temporary missing information in the deduction process.

In our model we do not express coordination in terms of rewriting rules; moreover, the logic layer is enhanced by considering several types of logic languages.

\section{Logic- and chemical-based coordination model}

\subsection{Definition of the model}

The chemical-based coordination model we present in this paper is designed to exploit several important features of the models cited above in the context of self-organising and self-adaptive applications; our goal is to define a coordination model with the following characteristics: (i) coordination algorithms can be described in an sufficiently abstract way starting from high-level specifications; (ii) the constructs used to express coordination algorithms are amenable to formal analysis of their correctness, they incentivize the decoupling of logic from implementation and they meet software engineering properties such as modularity, reusability and compactness. The rationale leading the definition of our coordination model can be synthesized as the adoption of Kowalski's terminology [12]: algorithm $=$ logic + control. This formulation promotes the dichotomy of algorithms in: (i) logic components (formulae) that determine the meaning of the algorithm, the knowledge used to solve a problem (i.e. what has to be done) and (ii) control components, which specify the manner the knowledge is used (i.e. how it has to be done).

The coordination model we define (Figure 1b) is a generalization of the SAPERE model with two additional features: (i) LSAs can store not only data tuples but actual logic programs (Section 3.2); (ii) the bonding eco-law is replaced by a new one named Logic eco-law, which is in charge of executing logic programs and performing the bonding actions. 


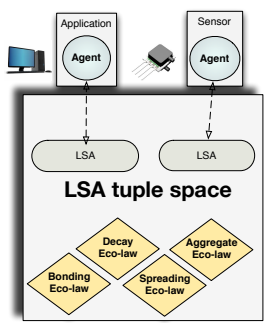

(a) SAPERE model

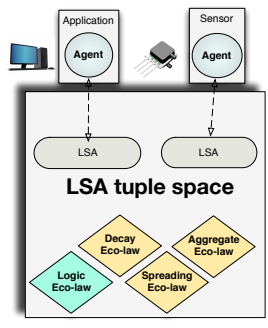

(b) Our model

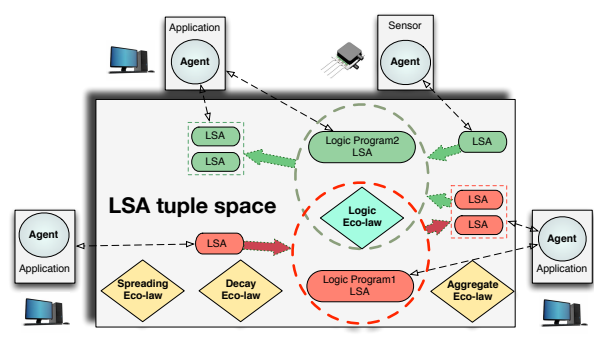

(c) Reactions among logic programs

Figure 1: The generalization of the SAPERE Model

The remaining components of the model are exactly the same as the ones of the SAPERE model. The virtual chemical reactions among tuples taking place in the shared container are now driven by logic inferences processes, which produce either data tuples or new logic programs during the "execution" of logic programs (Figure 1c). This process brings the idea promulgated by [12] in the context of chemical-based coordination models: the logic components of an algorithm are expressed in terms of logic programs, here embedded in LSAs, which can react among each other in a chemical fashion. Similarly, agents implement the control components (written in a programming language such as Java), and they perform computations according to the knowledge inferred by logic programs. This approach to separation and mapping of concepts helps designing coordination algorithms from an abstract point of view. On the one hand, algorithms are thought as interactions of atomic logic entities which define the meaning (in Kowalski's terminology) of subparts of the original algorithm. On the other hand, once logic entities have been defined, a specific problem-solving strategy can be chosen to be implemented for each subpart of the original problem. The intuition of using logic programs is twofold: (i) tuples exchanges represent the basic mechanism to carry out indirect communication among agents, thus the state and the evolution of a coordination process can be defined by analysing the set of tuples in the containers; (ii) tuples are used as inputs (facts) and produced as outputs of logic programs (models and formulae obtained by resolution rules). By considering points (i) and (ii), logic programs provide a natural formal tool to express coordination, allowing for inferred formulae to state relationships among entities of the system, depicting the evolution of coordination processes and proving system properties. 


\subsection{Logic programs}

Logic programs [14] are sets of logic formulae and are expressed in a logic language (e.g. first-order logic). Executing a logic program means either: (i) providing queries to the program and testing whether they logically follow from the program by using a proof engine (logic inference) or (ii) inferring all sentences that logically follow from the program (logic semantics). An interpretation of a formal language is an interpretation (see [14]) of constants, predicate and functions of the language over a given domain. The truth-value of a logic sentence is determined by the interpretation of the logic connectives. Given a logic program $P$, a model is an interpretation $M$ such that every formula in $P$ is true (depicted as $M \mid=P$ ). Here we are interested in Herbrand interpretations ([14]): (i) the implicit domain is the Herbrand Universe, the closure of the set of constants under all the functions symbols of the language; (ii) constants are interpreted as themselves and every function symbol as the function it refers to. In classical 2-valued logic programs, Herbrand interpretation can be defined through sets of atoms implicitly interpreted as true.

Example: $P=(C(x) \leftarrow A(x), B(x) ; \quad A(c) \leftarrow \square ; B(c) \leftarrow \square$; ) is a definite logic program [14]. Clauses are implicitly universally quantified. This is a definite logic program (i.e. containing Horn clauses): $x$ is a variable, $c$ is a constant and here they range over an (implicitly) defined domain. The first rule is composed of the head $C(X)$ and the body $A(X), B(X)$ and it can be read as "C $C(X)$ is true if both $A(X)$ and $B(X)$ are true". Rules with empty bodies $(\square)$ are named facts and they state sentences whose heads must be considered satisfied; in this case $A(c)$ and $B(c)$ hold. $M=\{A(c), B(c), C(c)\}$ is a model for the program in the example, because it satisfies all the rules.

\subsection{Logic languages}

In our model, logic programs are executed by the Logic eco-law. An important point in our approach is the generality of the coordination model w.r.t. the logic. We consider only logic languages that support Herbrand's interpretations, whereas we do not put any constraint on the inference methods or the semantics. Both inference methods and semantics are treated as parameters associated with logic programs. From the practical point of view, for each logic language we require the implementation of a dedicated Logic eco-law that executes the corresponding logic programs. This feature makes possible to use, possibly simultaneously: (i) 
several types of logic programs (e.g. definite, general logic programs, several types DATALOG or DATALOG-inspired programs) associated with two-valued, multi-valued (e.g. Belnap's logic) or paraconsistent logics; (ii) several inference procedures (e.g. $S L D, S L D N F$ ) and semantics (e.g. Aptvan Emden-Kowalski, Kripke-Kleen, stable,well-founded model semantics) $[11,13,1,9,17]$.

\subsection{Logic Fragments}

In our model, logic programs are embedded in logic units named Logic Fragments. The following set of definitions will be used to clarify the concept. We assume that Prop, Const and Var are finite mutually disjoint sets of relation symbols, constants and variables respectively. We will identify variables with letters $x, y, \ldots$ and constants with letters $a, b, \ldots$. Definition 1 (Literals, Ground Literals): A literal $\hat{P}$ is an expression of type $P\left(X_{1}, \ldots, X_{n}\right)$ or $\neg P\left(X_{1}, \ldots, X_{n}\right)$ where $P \in$ Prop and $X_{i} \in$ $($ Const $\cup$ Var $)$ for $i=1, \ldots, n$. A ground literal is a literal without variables. The set of all ground literals w.r.t. a set Const is denoted $G$ (Const). The power set of $G$ (Const) is depicted $\mathcal{P}(G)$.

Definition 2 (Valuations): A valuation $w$ is a function from $\operatorname{Var}$ to Const that assigns a constant $c_{i}$ to each variable $x_{i}$. The set of all possible valuations is depicted as $\mathcal{W}=\{w \mid w:$ Var $\rightarrow$ Const $\}$.

Definition 3 (Instances of Literal): If $\hat{P}$ is a literal and $w$ is a valuation, with $\hat{P}_{w}$ we identify the ground literal where every variable of $\hat{P}$ has been replaced by a constant according to the definition of $w . \hat{P}_{w}$ is named an instance of $\hat{P}$. We denote $I_{\hat{P}}=\left\{\hat{P}_{w} \mid w \in \mathcal{W}\right\} \subseteq G($ Const $)$.

Definition 4 (Logic Programs): A logic program is a set of logic formulae written in a logic language using: (i) literals $\hat{P}_{1}, \ldots, \hat{P}_{n}$ defined over Prop, Const, Var and (ii) logic operators.

Definition 5 (A-generator): Given a literal $P\left(X_{1}, \ldots, X_{n}\right)$, an A-generator w.r.t. a function $U:$ Const $^{n} \rightarrow\{T, F\}$ is the finite set:

$P^{U}\left(X_{1}, \ldots, X_{n}\right)=\left\{P\left(c_{1}, \ldots, c_{n}\right) \in I_{P\left(X_{1}, \ldots, X_{n}\right)} \mid U\left(c_{1}, \ldots, c_{n}\right)=T\right\}$.

Example: $A^{U}(X)=\{A(X) \mid X \in\{a, b, c\}\}=\{A(a), A(b), A(c)\}$, with $U(a)=U(b)=U(c)=T$.

Definition 6 (I-generator): Given a literal $P\left(X_{1}, \ldots, X_{n}\right)$, an I-generator w.r.t a function $V: \mathcal{P}(G) \rightarrow \mathcal{P}(G)$ and a finite set $H \subseteq \mathcal{P}(G)$ is the set: $P^{H, V}\left(X_{1}, \ldots, X_{n}\right)=\left\{P\left(c_{1}, \ldots, c_{n}\right) \in I_{P\left(X_{1}, \ldots, X_{n}\right)} \cap V(H)\right\}$

If $V$ is omitted, we assume that $V(H)=H$ (identity function).

Example: if $N=\{2,3,4\}$ and $V(N)=\{$ Even $(x) \mid x \in N \wedge x$ is even $\}$, then $\operatorname{Even}^{N, V}(X)=\{\operatorname{Even}(2), \operatorname{Even}(4)\}$. 
The rationale of such definitions is to provide the program with a set of facts built from conditions holding on tuples stored in the container. The unfolding of these generators produces new facts for the interpretation of the logic program.

By $\mathcal{L F}$ we identify the algebraic structure of Logic Fragments, recursively defined as follows:

Definition 7 (Logic Fragments $\mathcal{L F}$ ):

(I) $\triangle \in \mathcal{L F}$

(II) (Grouping) If $e \in \mathcal{L F}$ then $(e) \in \mathcal{L F}$

(III) (Parallel-and) If $e_{1}, e_{2} \in \mathcal{L F}$ then $e_{1} \sqcap e_{2} \in \mathcal{L F}$

(IV) (Parallel-or) If $e_{1}, e_{2} \in \mathcal{L F}$ then $e_{1} \sqcup e_{2} \in \mathcal{L F}$

(V) (Composition) If $P$ is a logic program, $\mathcal{M}$ an execution modality, $S$ a set of A,I-generators, $\varphi: \mathcal{P}(G) \rightarrow\{T, F\}$ and $e_{p} \in \mathcal{L F}$ then $\left(P, \mathcal{M}, e_{P}, S, \varphi\right) \in \mathcal{L F}$.

$\triangle$ is a special symbol used only in Logic Fragments to depict all the tuples in the container (both LSAs and Logic Fragments). $\mathcal{M}$ is the identifier of the way $P$ is "executed" (we will use $\mathcal{M}=\mathcal{A}$ for the Apt-van Emden-Kowalski and $\mathcal{M}=\mathcal{K}$ for the Kripke-Kleen semantics). $e_{P}$ is named constituent of the Logic Fragment and it is interpreted as a set of tuples used as support to generate the facts for the program. $S$ is a set of A,I-generators used to derive new facts from $P$. The function $\varphi: \mathcal{P}(G) \rightarrow\{T, F\}$ returns $T$ if the tuples represented by the constituent $e_{p}$ satisfy some constraints; the logic program is executed if and only $\varphi\left(e_{P}\right)=T$ (Def. 8). $\varphi_{T}$ is constant and equal to $T$. For style reason, we will write $P^{\mathcal{M}}\left(e_{P}, S, \varphi\right)$ instead of $\left(P, \mathcal{M}, e_{P}, S, \varphi\right)$.

Every Logic Fragment is executed by the Logic eco-law; its semantics is defined by using the function $v_{L}$.

Definition 8 (Semantic function): $v_{L}: \mathcal{L F} \rightarrow \mathcal{P}(G) \cup\{\bowtie\}$ associates the fragment with the set of tuples inferred by the logic program (consequent) or with $\bowtie$, which stands for undefined interpretation. $L$ denotes the set of actual tuples in the container before executing a Logic Fragment. Operators are ordered w.r.t. these priorities: grouping (highest priority), composition, $\sqcap$ and $\sqcup$ (lowest priority). $v_{L}$ is recursively defined as follows:

I) $v_{L}(\triangle) \triangleq L$

II) $v_{L}((e)) \triangleq v_{L}(e)$

III) $v_{L}\left(e_{1} \sqcap \ldots \sqcap e_{n}\right)_{n \geq 2} \triangleq \begin{cases}\bowtie & \text { if } \exists i \in\{1, \ldots, n\} \cdot v_{L}\left(e_{i}\right)=\bowtie \\ \bigcup_{i=1}^{n} v_{L}\left(e_{i}\right) & \text { otherwise }\end{cases}$

IV) $v_{L}\left(e_{1} \sqcup \ldots \sqcup e_{n}\right)_{n \geq 2} \triangleq \begin{cases}\bigcup_{i \in \mathcal{I}} v_{L}\left(e_{i}\right) & \text { if } \mathcal{I}=\left\{e_{i} \mid v_{L}\left(e_{i}\right) \neq \bowtie, 0 \leq i \leq n\right\} \neq \emptyset \\ \bowtie & \text { otherwise }\end{cases}$

V) $v_{L}\left(P^{\mathcal{M}}\left(e_{P}, S, \varphi\right)\right) \triangleq Q$ 
$Q$ is the consequent of $P^{\mathcal{M}}$ and it is defined as follows: if $\mathcal{M}$ is not compatible with the logic program $P$ or if $v_{L}\left(e_{p}\right)=\bowtie$ or if $\varphi\left(v_{L}\left(e_{p}\right)\right)=$ $F$ then $Q=\bowtie$. $\varphi$ "blocks" the execution of the program as long as a certain condition over $e_{p}$ is not satisfied. Otherwise, based on $S=$ $\left\{P_{0}^{H_{0}, V_{0}}\left(X_{01}, \ldots, X_{0 t_{0}}\right), \ldots, P_{n}^{H_{n}, V_{n}}\left(X_{n 1}, \ldots, X_{n t_{n}}\right), P_{0}\left(Y_{01}, \ldots, Y_{0 z_{0}}\right), \ldots\right.$ $\left.P_{m}\left(Y_{m 1}, \ldots, Y_{m z_{m}}\right)\right\}$, the Logic eco-law produces the set of facts $F s=$ $\bigcup_{i=0}^{n} P_{i}^{v_{L}\left(H_{i}\right), V_{i}}\left(X_{i 1}, \ldots, X_{i t_{i}}\right) \cup \bigcup_{i=0}^{m} P_{i}\left(Y_{i 1}, \ldots, Y_{i z_{i}}\right)$. A,I-generators are then used to define sets of ground literals for the logic program which satisfy specific constraints; during the evaluation, for every set $H_{i}$ we have either $H_{i}=e_{p}$ or $H_{i}=\triangle . Q$ is finally defined as the set of atoms inferred by applying $\mathcal{M}$ on the new logic program $P^{\prime}=P \cup\{l \leftarrow \square \mid l \in F s\}$, enriched by all the facts contained in $F s$. Note that there may be no need to explicitly calculate all the literals of A,I-generators beforehand: the membership of literals to generators sets may be tested one literal at a time or skipped because of the short-circuit evaluation.

Lemma 1 (Properties of operators): Given $a, b \in \mathcal{L} \mathcal{F}$ with $a \equiv b$ we state that $v_{L}(a)=v_{L}(b)$ for every set of literals $L$. Then for any $a, b, c \in \mathcal{L F}$ :

I)
(Idempotence of $\sqcup$ )

(Commutativity of $\sqcup$ )

(Associativity of $\sqcup$ )

(Idempotence of $\sqcap$ )

(Commutativity of $\sqcap$ )

(Associativity of $\sqcap$ )

VII) $a \sqcap(b \sqcup c) \equiv(a \sqcap b) \sqcup(a \sqcap c) \equiv(b \sqcup c) \sqcap a$ (Distrib. of $\sqcap$ over $\sqcup)$

Intuitively, composing two Logic Fragments means calculating the inner one first and considering it as constituent for the computation of the second one. Parallel-and $(\sqcap)$ means executing all the Logic Fragments them in a row or none, whereas Parallel-or $(\sqcup)$ means executing only those ones that can be executed at a given time.

\subsection{Update of the container}

In our model, all the Logic Fragments are carried on a snapshot image of the container, i.e. given a Logic Fragment $e$ in the container, if $v_{L}(e) \neq \bowtie$, then it is evaluated as an atomic operation (every symbol $\triangle$ in the sub Logic Fragments which composes $e$ is always translated with the same set of actual tuples). Multiple Logic Fragments ready to be evaluated are computed in a non-deterministic order. The tuples inferred by the logic programs (with all used facts) are inserted in the container only when the evaluation of the whole logic program terminates. At that point, the Logic 
eco-law injects the inferred tuples in the container and notifies the end of inference process to the agent. The Logic Fragment is subject to a new evaluation process as soon as the set $F s$ changes due to updates of the shared container, but there are no concurrent parallel evaluations of the same Logic Fragment at a given time (unless it appears twice); this aspect can potentially hide tuples updates in the evaluation process (Section 5). The representation of the functions associated with A,I-generators depends on the implementation.

\section{Case studies}

By using Logic Fragments we can easily tackle interesting coordination problems and properties. Additional examples are reported in [6].

\subsection{Palindrome recognition}

As a first example we show an easy pattern recognition scenario. Assuming that an agent $A$ inserts positive integers into the container, we want to discover which ones are palindromic numbers (i.e. numbers that can be read in the same way from left to right and from right to left). We assume that these integers are represented by tuples of type $N(a)$, where $a$ is a number, e.g. $N(3)$ represents the number 3. Agent $A$ inserts the Logic Fragment $L F_{p}: P_{p}^{\mathcal{A}}\left(\triangle,\left\{N^{\triangle}\right.\right.$, TestPalin $\left.\}, \varphi_{p}\right)$.

$$
\begin{aligned}
& \varphi_{p}(\triangle)=T \Leftrightarrow \exists w: N(X)_{w} \in \triangle \\
& \text { TestPalin }(x)=\left\{\text { TestPalin }(a) \mid a \text { is a positive palindromic number less than } d_{\max }\right\}
\end{aligned}
$$

\section{Logic code 1.1 Definite logic program $P_{p}$}

$\operatorname{Palin}(x) \leftarrow N(x)$, TestPalin $(x)$

$P_{p}$ is the logic program in Code 1.1, evaluated with the Apt-van Embden Kowalski semantics $(\mathcal{A})$. The set $S$ of A,I-generators is composed of two elements: $N^{\triangle}$ contains all literals $N(a)$ (numbers) existing in the container $(\triangle)$; TestPalin $(x)$ contains all the literals of type TestPalin $(a)$, where $a$ is a positive palindromic number less then $d_{\max }$. These two sets of literals are treated as facts for $P_{p}$. According to $\varphi, P_{p}$ is executed as soon as a number $N(a)$ is inserted into the container. The rule of the logic program $P_{p}$ states that a number $a$ is a palindromic number $(\operatorname{Palin}(a))$ if $a$ is a number $(N(a))$ and $a$ passes the test for being palindromic (TestPalin $(a))$. We consider the tuple space shown in Figure $2 \mathrm{a}$ and $2 \mathrm{~b}$. At the beginning, agent $A$ injects $L F_{p}$ (Figure 2a). At a 
later stage $A$ injects $N(22)$ and the Logic Fragment is then executed. In this case, $N^{\triangle}$ is evaluated as $\{N(22)\}$. Moreover, TestPalin $(a)$ will contain TestPalin(22), because it is palindromic. This means the consequent $Q$ of $L F_{p}$ contains Palin(22), along with all the facts generated by the A,I-generators used in the logic program. If now agent $A$ injects $N(12)$, the Logic Fragment is re-executed and $N^{\triangle}$ is evaluated as $\{N(22), N(12)\}$. This second number does not satisfy the palindromic test $(N(12) \notin$ TestPalin $(x))$, so the 12 will not be considered as palindromic. Finally $A$ injects $N(414)$ and during the re-execution of $L F_{p}$ we obtain: $N^{\triangle}=\{N(22), N(12), N(414)\}$ and $N(414) \in$ TestPalin $(x)$, so the consequent $Q$ will contain Palin(22) and Palin(414) (Figure 2b). Note that if numbers were injected by agents different from $A$ (like a sensor), the same reactions would take place.

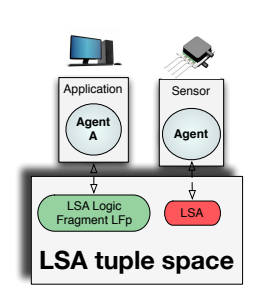

(a) Injection of $L F_{p}$

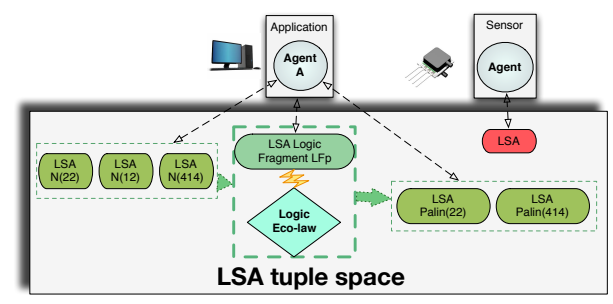

(b) Injection of numbers

Figure 2: Evolution of the container for the example of Section 4.1

Property 1: A palindromic integer $a \geq 0$ exists in the container if and only if Palin ( $a$ ) exists in the least Herbrand model of $P_{p}^{\prime}$ (the extension of $P_{p}$ with all the facts created by A,I-generators).

Proof sketch: The property above states that by using the Logic Fragment $L F_{p}$ we are able to correctly find out all the palindromic integers. Thanks to the logic programs and the semantic of Logic Fragments, we can easily verify that if such integers exist in the container then their literals are inferred in Herbrand model of $P_{p}^{\prime}$. Moreover, given that such literals are only generated by $L F_{p}$, if such literals exist in the model then there must be the associated palindromic integers in the shared space.

\subsection{Gradient and Chemotaxis patterns - general programs}

In this second example we use Logic Fragments to implement the gradient and chemotaxis design patterns ([10]), which are two bio-inspired mechanisms used to build and follow shortest paths among nodes in a network. 
The chemotaxis is based on the gradient pattern. A gradient is a message spread from one source to all the nodes in the network, carrying a notion of distance from the source (hop-counter). Gradient messages can also carry user-information. Once a node receives a gradient from a known source whose hop-counter is less than the local one (i.e. a new local shortest-path has been found), the node updates its local copy of the hopcounter (aggregation) and spreads it towards the remaining neighbours with a hop-counter incremented by one unit. In these terms, the gradient algorithm is similar to the distance-vector algorithm. The chemotaxis pattern resorts to gradient shortest-paths to route messages towards the source of the gradient. We can implement the gradient and chemotaxis patterns by using an agent $A_{g c}$ associated with the Logic Fragment:

$$
L F_{g c}: P_{g}^{\mathcal{A}}\left(P_{a}^{\mathcal{A}}\left(\triangle \sqcap P_{n}^{\mathcal{K}}\left(\triangle, S_{n}, \varphi_{n}\right), S_{a}, \varphi_{T}\right), S_{g}, \varphi_{T}\right) \sqcup P_{c h}^{\mathcal{A}}\left(\triangle, S_{c h}, \varphi_{c h}\right)
$$

\section{Logic code 1.2 Program $P_{n}$ - Next hop initialization}

$\operatorname{GPath}\left(\mathrm{x}, d_{\max }\right.$, null $) \leftarrow \neg$ existsGPath $(\mathrm{x})$

Logic code 1.3 Program $P_{a}$ - Aggregation

$\operatorname{cmpGradient}\left(x_{1}, x_{2}, y_{1}, y_{2}, z\right) \leftarrow \operatorname{Gmsg}\left(x_{1}, x_{2}, y_{1}, z\right), \operatorname{GPath}\left(x_{1}, y_{2}, w\right)$

updateGPath $\left(x_{1}, y_{1}, x_{2}, z\right) \leftarrow \operatorname{cmpGradient}\left(x_{1}, x_{2}, y_{1}, y_{2}, z\right), \operatorname{less}\left(y_{1}, y_{2}\right)$

Logic code 1.4 Program $P_{g}$ - Spreading

$\operatorname{spreadGradient}\left(x_{1}, \operatorname{local}, z, y, x_{2}\right) \leftarrow \operatorname{updateGPath}\left(x_{1}, y, x_{2}, z\right)$

Logic code 1.5 Program $P_{c h}$ - Chemotaxis

$\operatorname{sendChemo}(m, x, w) \leftarrow \operatorname{Cmsg}(m, x), \operatorname{GPath}(x, y, w)$

$\varphi_{n}(\triangle)=T \Leftrightarrow \exists w: \operatorname{Gmsg}\left(x_{1}, x_{2}, y, z\right)_{w} \in \triangle, \quad \varphi_{c h}(\triangle)=T \Leftrightarrow \exists w: C m s g(x, y)_{w} \in \triangle$

$S_{c h}=\left\{\mathrm{Cmsg}^{\triangle}\right.$, GPath $\left.^{\triangle}\right\} S_{g}=\left\{\right.$ updateGPath $\left.^{e_{P_{g}}}\right\} \quad S_{n}=\left\{\right.$ existsGPath $^{\triangle, V}$, Gmsg $\left.^{\triangle}\right\}$

$S_{a}=\left\{G m s g^{e_{P_{a}}}\right.$, GPath $^{e_{P_{a}}}$, less $\} \operatorname{less}(x, y)=\left\{\operatorname{less}(a, b) \mid a<b, a, b \in\left\{1, \ldots, d_{\text {max }}\right\}\right\}$

existsGPath $(x)^{\triangle, V}=\left\{\neg\right.$ existsGPath $\left.(a) \in I_{\neg \text { existsGPath }(x)} \cap V(\triangle)\right\}$

$V(\triangle)=\left\{\neg\right.$ existsGPath $\left.(a) \mid \exists w: G m s g(a, x, y, z)_{w} \in \triangle \wedge \neg \exists \operatorname{GPath}(a, y, w)_{w} \in \triangle\right\}$

Gradients are represented by tuples $\operatorname{Gmsg}(a, b, c, d)$ where $a$ is the ID of the source, $b$ is the ID of the last sender of the gradient, $c$ is the hopcounter and $d$ is the content of the message. Values null, $d_{\text {max }}$ and local are considered constants. Local hop-counter are stored in tuples of type $G P a t h(a, c, e)$, where $a$ and $c$ are as above and $e$ is the previous node in the 
path, this will be used to route the chemotaxis message downhill towards the source. $L F_{g c}$ is composed of several Logic Fragments; the parallel-or operator makes the agent $A_{g c}$ to react simultaneously to chemotaxis and gradients messages. The innermost fragment $e_{P_{a}}=\triangle \sqcap P_{n}^{\mathcal{K}}\left(\triangle, S_{n}, \varphi_{n}\right)$ is executed when a gradient message is received from a neighbour $(\triangle$ can be executed directly but the parallel-and operator blocks the execution of outer fragments until $P_{n}^{\mathcal{K}}\left(\triangle, S_{n}, \varphi_{n}\right)$ finishes); it initializes the GPath tuple for the source of the gradient. By using the composition operator, the literals inferred in the model of $P_{n}^{\prime}$, along with all the tuples in the container (fragment $\triangle$ ) are then treated as constituent for the fragment $e_{P_{a}}=P_{a}^{\mathcal{A}}\left(e_{P_{e}}, S_{a}, \varphi_{T}\right)$, i.e. they are used to generate facts for the program $P_{a}^{\prime}$. This one is used to aggregate the hop-counter for the source with the one stored in the local container. $e_{P_{a}}$ is finally treated as constituent for the fragment $e_{P_{g}}=P_{g}^{\mathcal{A}}\left(e_{P_{a}}, S_{g}, \varphi_{T}\right)$. Note that aggregation happens before spreading, imposing an order on the reactions. $P_{g}^{\mathcal{A}}$ is used to verify whether the gradient message must be spread to the neighbours. If so, a literal spreadGradient ( $a$, local $, d, c, b)$ is inferred during the computation of its semantics, where local is translated with the name of the current node. Simultaneously, the Logic Fragment $P_{c h}^{\mathcal{A}}\left(\triangle, S_{c h}, \varphi_{c h}\right)$ is executed as soon as a chemotaxis message is received (described as $C m s g(f, g)$, with $f$ content of the message and $g$ ID of the receiver). That Logic Fragment uses the local copy of the hop-counter to infer which is the next hop to which the chemotaxis message must be sent to (relay node). If the local hop-counter exists, a literal sendChemo $(f, g, h)$ is generated in the model of $P_{c h}^{\prime}$, with $h$ representing the ID of the next receiver of the chemotaxis message. Otherwise, the message remains in the container until such a literal is finally inferred. All the literals contained in the consequent $Q$ of $L F_{g c}$ are used by the agent $A_{g c}$ to manage the control part of the algorithm, described in the following code.

Control code 1.6 Behaviour of agent $A_{g c}$

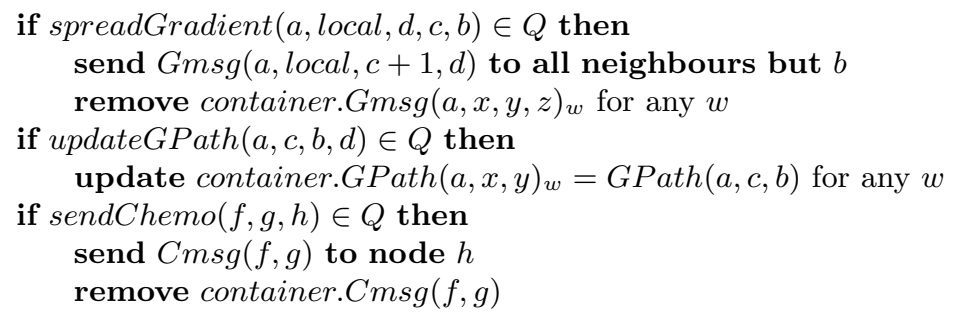

We consider the network of Figure 3; the Logic Fragment can be used to provide the gradient and chemotaxis functionalities as services to other 
agents running on the same nodes. Assuming that agent $A_{G m}$ on node $A$ wants to send a query message $m_{1}$ to all the nodes of the network, it creates and injects the gradient message $\operatorname{Gmsg}\left(A, A, 0, m_{1}\right)$. At this point a reaction with $L F_{g c}$ takes place, generating in the consequent $Q$ of $L F_{g c}$ literals $\operatorname{GPath}(A, 0, A)$ (semantics of $\left.P_{n}^{\prime}\right)$ and $\operatorname{spreadGradient}\left(A, A, m_{1}, 0, A\right)$ (semantics of $P_{g}^{\prime}$ ). The second literal causes the spreading of the gradient message to nodes $B$ and $C$. Similar reactions take place in the remaining nodes. If we assume that the gradient passed by node $D$ is the first one to reach $E$ then $\operatorname{GPath}(A, 3, D)$ is inferred in the consequent $Q$ on node $E$. When the gradient message coming from $B$ reaches $E$, updateGPath $\left(A, 1, B, m_{1}\right)$ is inferred in the semantics of program $P_{a}^{\prime}$, so the hop-counter tuple is updated in $\operatorname{GPath}(A, 2, B)$. Now assuming that agent $A_{C m}$ on node $E$ wants to send a reply-message $m_{2}$ to node $A$, it creates and injects a chemotaxis message $C m s g\left(m_{2}, A\right)$. On the basis of the tuple $\operatorname{GPath}(A, 2, C)$, the literal sendChemo $\left(m_{2}, A, C\right)$ is inferred in the model of $P_{g}^{\prime}$, so the message is sent to node $B$. Similar reactions take place on node $B$, which finally sends the chemotaxis message to node $A$.

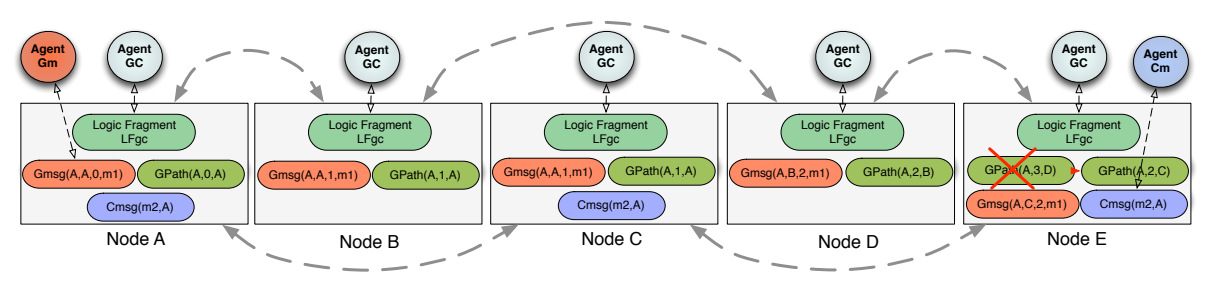

Figure 3: Network of 5 nodes

Property 2: Let $\mathcal{N}$ be a network with no disconnected hosts. If we assume that: (i) nodes do not move; (ii) every node has a Logic Fragment of type $L F_{g c}$; (iii) every information sent from one node to another one arrives at destination in a finite time (eventually due to multiple spreading); (iv) a gradient message is created by an agent $A_{G m}$ on one node $S$ of $\mathcal{N}$, then there exists a finite time $t^{*}$ for which the following statement holds: if an agent $A_{C m}$ on node $R$ creates a chemotaxis message for $A$ at time $t>t^{*}$, then the chemotaxis message reaches the destination $S$ following a shortest-path between $R$ and $S$.

Proof sketch: The rationale behind the proof consists in proving two categories of properties: (i) a local property which states that the number of gradient messages sent by each node is finite, due to the decrements of 
the hop-counter caused by the applications of the aggregation-function; (ii) global properties, based on the local property holding in each node (e.g. we prove the creation of the shortest-path). The details are reported in [6]. Additional studies focusing on the integration of spatial-temporal logics in Logic Fragment are needed to prove the analogous statement when considering mobile nodes.

\section{Conclusion and Future works}

In this paper we have presented a chemical-based coordination model based on a logic framework. Virtual chemical reactions are lead by logic deductions, implemented in terms of combination of logic programs. This approach combines the benefits of using a chemical-based coordination model along with the expressiveness of several distinct types of logic languages to formalise coordination logically. Intuitively, even though no formal verification or validation methods were presented, the rationale behind the proof of the correctness of coordination algorithm follows from a formalisation of the system properties to be proved in terms of logical formulae. This paves the way for at least two formal analysis: (i) what-if assessment - coordination events can be modeled in terms of injected/removed tuples and deducted literals can be used to test the satisfaction of the system properties formulae. This first kind of verification can be done at design time, to assess properties of the whole system under certain conditions (events) and partially at run-time, to infer how the system will evolve assuming a knowledge restricted to a certain subset of locally perceived events; (ii) the second type of design time analysis starts from the literals that satisfy the properties formulae and proceeds backwards, to derive what are the events that lead the system to that given state. Future works will focus on such aspects, to derive formal procedures for correctness verification of algorithm built on top of Logic Fragments.

Several kinds of logics present interesting features to model and validate coordination primitives: (i) paraconsistent logics (e.g. [17]) and (ii) spatial-temporal logics, to assert properties depending on location and time parameters of system components. We plan also to realise an implementation of the model, including several semantics for Logic Fragments taking inspiration from the coordination primitives presented in [7].

\section{References}

1. Apt, K.R., van Emden, M.H.: Contributions to the theory of logic programming. J. ACM 29(3), 841-862 (1982) 
2. Banâtre, J.P., Le Métayer, D.: The gamma model and its discipline of programming. Sci. Comput. Program. 15(1), 55-77 (1990)

3. Berry, G., Boudol, G.: The chemical abstract machine. In: Proceedings of the 17th ACM SIGPLAN-SIGACT Symposium on Principles of Programming Languages. pp. 81-94. POPL '90, ACM (1990)

4. Castelli, G., Mamei, M., Rosi, A., Zambonelli, F.: Pervasive middleware goes social: The sapere approach. In: Proceedings of the 2011 Fifth IEEE Conference on SelfAdaptive and Self-Organizing Systems Workshops. pp. 9-14. SASOW '11 (2011)

5. Ciancarini, P., Franzè, F., Mascolo, C.: A coordination model to specify systems including mobile agents. In: Proceedings of the 9th International Workshop on Software Specification and Design. IWSSD '98, IEEE Computer Society, Washington, DC, USA (1998)

6. De Angelis, F.L., Di Marzo Serugendo, G.: Towards a logic and chemical based coordination model (2015), https://archive-ouverte.unige.ch/

7. Denti, E., Natali, A., Omicini, A., Venuti, M.: Logic tuple spaces for the coordination of heterogeneous agents. In: Baader, F., Schulz, K.U. (eds.) Frontiers of Combining Systems, Applied Logic Series, vol. 3, pp. 147-160. Kluwer Academic Publishers (1996), 1st International Workshop (FroCoS'96), Munich, Germany, 26-29 Mar. 1996. Proceedings

8. Dershowitz, N., Manna, Z.: Proving termination with multiset orderings. Commun. ACM 22(8) (1979)

9. Emden, M.H.V., Kowalski, R.A.: The semantics of predicate logic as a programming language. Journal of the ACM 23, 569-574 (1976)

10. Fernandez-Marquez, J.L., Di Marzo Serugendo, G., Montagna, S., Viroli, M., Arcos, J.L.: Description and composition of bio-inspired design patterns: a complete overview. Natural Computing 12(1), 43-67 (2013)

11. Fitting, M.: Fixpoint semantics for logic programming a survey. Theoretical Computer Science 278(1-2), 25 - 51 (2002), mathematical Foundations of Programming Semantics 1996

12. Kowalski, R.: Algorithm = logic + control. Commun. ACM 22(7), 424-436 (Jul 1979)

13. Kowalski, R., Kuehner, D.: Linear Resolution with Selection Function. Artificial Intelligence 2(3-4), 227-260 (Dec 1971)

14. Nilsson, U., Maluszynski, J.: Logic, Programming, and PROLOG. John Wiley \& Sons, Inc., New York, NY, USA, 2nd edn. (1995)

15. Omicini, A., Denti, E.: From tuple spaces to tuple centres. Science of Computer Programming 41(3), 277-294 (nov 2001)

16. Viroli, M., Casadei, M., Omicini, A.: A framework for modelling and implementing self-organising coordination. In: Shin, S.Y., Ossowski, S., Menezes, R., Viroli, M. (eds.) 24th Annual ACM Symposium on Applied Computing (SAC 2009). vol. III, pp. 1353-1360. ACM, Honolulu, Hawai'i, USA (8-12 Mar 2009)

17. Vitória, A., Maluszyński, J., Szałas, A.: Modeling and reasoning in paraconsistent rough sets. Fundamenta Informaticae 97(4), 405-438 (2009)

18. Zambonelli, F., Omicini, A., Anzengruber, B., Castelli, G., De Angelis, F.L., Di Marzo Serugendo, G., Dobson, S., Fernandez-Marquez, J.L., Ferscha, A., Mamei, M., Mariani, S., Molesini, A., Montagna, S., Nieminen, J., Pianini, D., Risoldi, M., Rosi, A., Stevenson, G., Viroli, M., Ye, J.: Developing pervasive multiagent systems with nature-inspired coordination. Pervasive and Mobile Computing 17, 236-252 (2015), special Issue 10 years of Pervasive Computing In Honor of Chatschik Bisdikian 